\title{
The role and possible mechanism of IncRNA AC092159.2 in modulating adipocyte differentiation
}

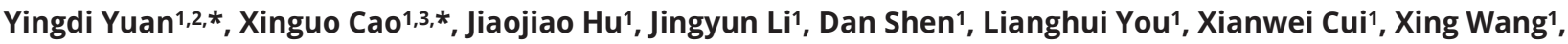

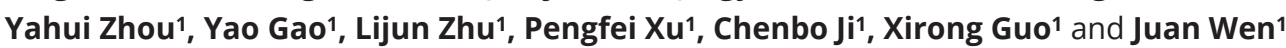 \\ ${ }^{1}$ Nanjing Maternity and Child Health Care Institute, Women's Hospital of Nanjing Medical University, Nanjing Maternity and Child Health Care Hospital, \\ Nanjing, China \\ 2Department of Pediatrics, The First People's Hospital of Lianyungang, Xuzhou Medical University Affiliated Hospital of Lianyungang (Lianyungang Clinical \\ College of Nanjing Medical University), Lianyungang, China \\ ${ }^{3}$ Cancer Care Ontario, Toronto, Canada
}

Correspondence should be addressed to X Guo or J Wen: xrguo@njmu.edu.cn or wenj2010@gmail.com

*Y Yuan and X Cao contributed equally to this work

\begin{abstract}
Obesity is a major risk factor for metabolic diseases, while adipocyte differentiation is closely related to obesity occurrence. Long noncoding RNAs (IncRNAs) are a unique class of transcripts in regulation of various biological processes. Using IncRNA microarray, we found IncRNA AC092159.2 was highly expressed in differentiated HPA-v and located 247 bp upstream of the TMEM18, which was associated with BMI and obesity. We aimed to explore the role of AC092159.2 in adipogenesis and the underlying mechanisms. The effects of AC092159.2 gain- and loss-of-function on HPA-v adipogenesis were determined with lentivirus and siRNA-mediated cell transduction, respectively. Lipid accumulation was evaluated by oil red O staining; the expression of AC092159.2, TMEM18 and several adipogenesis makers in HPA-v were analyzed by qPCR/Western blot. We found that the expression of AC092159.2 gradually increased during HPA-v differentiation, and its expression in omental adipose tissue was positively related with BMI among 48 human subjects. Overexpression of AC092159.2 promoted adipocytes differentiation while knockdown of it led to an adipogenic defect. Moreover, the expression of AC092159.2 and TMEM18 were positively correlated during adipogenic differentiation. AC092159.2 overexpression boosted TMEM18 expression while AC092159.2 knockdown restrained TMEM18 expression. Further rescue experiments showed that TMEM18 knockdown partially restrained adipogenic differentiation in AC092159.2 overexpressed HPA-V and adipogenic defect caused by AC092159.2 knockdown could be rescued by TMEM18 overexpression. Luciferase reporter assays revealed that AC092159.2 had a transcriptional activation effect on TMEM18. We concluded that IncRNA AC092159.2 promoted human adipocytes differentiation possibly by regulating TMEM18.
\end{abstract}

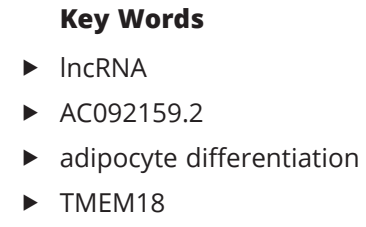

Journal of Molecular Endocrinology (2019) 62, 137-148 (c) 2019 Society for Endocrinology Published by Bioscientifica Ltd. Printed in Great Britain 


\section{Introduction}

Obesity refers to excessive accumulation of adipose tissue caused by genetic and environmental factors, which can significantly increase the morbidity and mortality of cardiovascular disease, type 2 diabetes, hyperlipidemia and malignancy (Haslam \& James 2005, Barth 2011, Serra-Majem \& Bautista-Castano 2013). What's more, it is growing with alarming speed and is becoming a global public health crisis ( $\mathrm{Ng}$ et al. 2014). Adipocyte differentiation is a vital process for adipocyte and development of obesity (Lu et al. 2012). Therefore, it is of great significance to study the molecular mechanism of adipocyte differentiation in the prevention and treatment of obesity and other adipocyte differentiation-related disorders. The mechanism of adipogenesis is extremely complex. At present, transcription factors and signal pathway of adipogenesis have been revealed to involve an elaborate network of transcriptional regulation that coordinates the expression of hundreds of proteins (Farmer 2006, Lefterova \& Lazar 2009, Cristancho \& Lazar 2011). Nevertheless, the detailed molecular mechanism of adipogenesis is not yet known.

LncRNAs are a unique class of transcripts that have regulatory roles in biological processes, such as cellular differentiation ( $\mathrm{Hu}$ et al. 2012, Rinn \& Chang 2012), transcriptional repression (Batista \& Chang 2013), cancer metastasis (Gupta et al. 2010), X chromosomal inactivation (Lee 2009) and chromatin remodeling (Tsai et al. 2010). Recently, with the help of chip technology and high-throughput RNA sequencing, IncRNAs have been found to be widely and differentially expressed in adipose tissue (Kornfeld \& Bruning 2014, Zhao \& Lin 2015, Huang et al. 2016, Yang et al. 2016). Although several lncRNAs (U90926, ADINR and Uc.417) have been reported to exert important roles during adipocyte differentiation (Xiao et al. 2015, Cui et al. 2016, Chen et al. 2017), the exact molecular mechanisms involved in lncRNA-mediated modulation of adipogenesis still remains unclear.

Human visceral preadipocytes (HPA-v) are commonly used to study adipocyte differentiation in vitro (BarbosaDesongles et al. 2013, Davidge-Pitts et al. 2014, Fujita et al. 2016). We used this system to identify the IncRNAs related to adipogenesis by IncRNA microarray, and found that a long noncoding RNA (lncRNA), AC092159.2, was highly expressed in differentiated HPA-v compared with undifferentiated HPA-v. Therefore, we hypothesized that IncRNA AC092159.2 may be possibly related to adipogenesis. To verify our hypothesis, we applied AC092159.2 lentivirus and siRNA mediated gain- or loss-of-function experiments and rescue experiments in HPA-v to explore the role and possible mechanism of lncRNA AC092159.2 in modulating adipocyte differentiation.

\section{Materials and methods}

\section{Human subjects}

Omental adipose tissues were prospectively collected from patients undergoing surgery for abdominal disorders. All of the patients had no endocrine disease, malignancy or severe systemic illness. According to the Working Group on Obesity in China (WGOC) in 2003 (Wang et al. 2007), subjects with $\mathrm{BMI} \geq 24 \mathrm{~kg} / \mathrm{m}^{2}$ were defined as overweight. As a result, 31 subjects with overweight/obesity and 17 controls with normal weight consented to participate in this study. Written informed consent was obtained from all participants. The study was approved by the institutional review board of Nanjing Maternity and Child Health Care Institute and the methods were carried out in accordance with the approved guidelines.

\section{Cell culture and adipocyte differentiation}

HPA-v cells (ScienCell Research Laboratories, San Diego, CA, USA) were maintained in preadipocyte medium (PAM; ScienCell Research Laboratories) supplemented with 5\% fetal bovine serum (FBS; Gibco Life Technologies), 1\% penicillin/streptomycin solution (P/S; ScienCell Research Laboratories) and $1 \%$ preadipocyte growth supplement (PAGS; ScienCell Research Laboratories) at $37^{\circ} \mathrm{C}$ in $5 \%$ $\mathrm{CO}_{2}$. To induce differentiation, fully confluent HPA-v (day 0 ) were incubated in a differentiation cocktail of serumfree PAM, supplemented with $250 \mathrm{nM}$ insulin, $1 \mathrm{mM}$ dexamethasone, $\quad 0.5 \mathrm{mM}$ 3-isobutyl-1-methylxanthine and $1 \mu \mathrm{M}$ rosiglitazone. The culture medium was replaced 4 days later. Then, cells were incubated in serum-free DMEM containing $250 \mathrm{nM}$ insulin, which were replaced every 2 days until lipid accumulated in the cells (Day 10). HPA-v cells were harvested on days $0,1,4,7$ and 10 for further investigations.

\section{Lentivirus and siRNA-mediated cell transduction}

Lentivirus overexpressing vectors, including the negative control (Lv-NC), AC092159.2 overexpressing lentivirus (Lv-AC092159.2) and TMEM18 overexpressing lentivirus (Lv-TMEM18) were purchased from GenePharma Inc (Shanghai, China) and transfected according to the 
manufacturer's instructions. When reaching about 40\% confluence, at the stage with the optimal infection efficiency, the preadipocytes were infected with $4 \mu \mathrm{L}$ of Lv-NC(viraltiter, $1 \times 10^{8} \mathrm{TU} / \mathrm{mL}$ ), Lv-AC092159.2 (viraltiter, $1 \times 10^{8} \mathrm{TU} / \mathrm{mL}$ ) or Lv-TMEM18 (viral titer, $1 \times 10^{8} \mathrm{TU} / \mathrm{mL}$ ) in $2 \mathrm{~mL}$ PAM culture medium mixed with $5 \mu \mathrm{g} / \mathrm{mL}$ Polybrene (Sigma-Aldrich). After $24 \mathrm{~h}$ of infection, fresh medium was added into the preadipocytes. The overexpression efficiency was checked by GFP expression using fluorescence microscopy and confirmed by real-time quantitative PCR (qPCR). After the infected cells reached confluency, differentiation was induced as described previously. For knockdown, cells at $60 \%$ confluence were transfected with AC092159.2 or TMEM18-specific small interfering RNA (siRNA) pool (Supplementary Table 1, see section on supplementary data given at the end of this article) using Lipofectamine 2000 (Invitrogen) at $100 \mathrm{nM}$ in OptiMEM medium (Thermo Fisher Scientific) according to the manufacturer's instruction. Control cells were treated with Stealth RNAi Negative Control (Thermo Fisher Scientific). And knockdown efficiency was confirmed by qPCR.

\section{Oil Red $\mathrm{O}$ staining for lipid and triacylglycerol (TG) content measurement}

In vitro differentiated cells at days 7 and 10 were purged with PBS twice and afterward fixed in $4 \%$ formaldehyde for $20 \mathrm{~min}$. After being rinsed by PBS twice, the fixed cells were stained with $0.2 \%$ Oil Red O (Sigma) for $60 \mathrm{~min}$ at $37^{\circ} \mathrm{C}$. Subsequently the stained cells were purged three times with distilled water and visualized under the microscope Observer D1 (Carl Zeiss). The content of intracellular triglyceride (TG) that is relative to total protein content was measured using the triglyceride assay kit (Applygen Technologies Inc, Beijing, China) and BCA Protein Assay Kit (Pierce) according to the manufacturer's instructions. At the indicated time, the mature adipocytes were treated with lysis buffer and the cell lysates were harvested and homogenized. Subsequently, the cell suspension was retained to respectively detect the triglyceride and protein concentration.

\section{RNA extraction and $q P C R$}

The total RNA was isolated from HPA-v cells using TRIzol (Thermo Fisher Scientific). The high-capacity cDNA reverse transcription kit (RR047A; Takara) was used for reverse transcription of normalized RNA, and cDNA was analyzed by qPCR using the SYBR Green method. Data were normalized to PPIA and further analyzed using the $2-\Delta \Delta \mathrm{Ct}$ method. All the primer sequences for the marker genes involved in the test were listed in Supplementary Table 2.

\section{Protein extraction and Western blot}

The protein was extracted from the differentiated adipocytes (day 7) for Western blot analysis using RIPA buffer and quantified by the BCA Protein Assay Kit (23229; Thermo Fisher Scientific). A total of $10 \mathrm{mg}$ protein was separated by $10 \%$ SDS-PAGE and transferred to PVDF membranes. Membranes were immunoblotted with specific primary antibodies against rabbit polyclonal- $\beta$ actin (Cell Signaling Technology, No.84575, dilution in 1:5000), rabbit polyclonal TMEM18 (Abcam, No.106597, dilution in 1:1000), rabbit polyclonal FABP4 (Abcam, No.66682, dilution in 1:1000), monoclonal rabbit PPAR $\gamma$ (Cell Signaling Technology, No. 2435S, dilution in 1:1000), rabbit polyclonal C/EBP $\alpha$ (Cell Signaling Technology, No.2295S, dilution in 1:1000) and polyclonal rabbit C/EBP $\beta$ (Cell Signaling Technology, No.3087S, dilution in 1:1000). The secondary antibody was horseradish peroxidaseconjugated goat anti-rabbit IgG (dilution in 1:5000) from Beijing Zhong Shan Biotechnology CO (Beijing, China).

\section{Cell proliferation assay}

The proliferation rates of the AC092159.2 overexpression and knockdown cells were measured using a WST-8 Cell Counting Kit-8 (CCK-8; Dojindo Molecular Technologies, Tokyo, Japan). Briefly, 3000 cells were seeded into each well of the 96-well plates. The media was replaced with an equal volume of fresh media containing 10\% CCK-8 after $0,24,48$ and $72 \mathrm{~h}$ of growth, and the cultures were incubated at $37^{\circ} \mathrm{C}$ for $1 \mathrm{~h}$. Cell growth was measured by microplate reader (Thermo Fisher Scientific) at an optical density (OD) of $450 \mathrm{~nm}$. The results are expressed as the mean \pm standard deviation (s.D.) of three separate experiments, with six determinations per experiment for each experimental condition.

\section{Flow cytometry assay}

The cell cycle and apoptosis of transfected cells were analyzed by flow cytometry. After transfection for $72 \mathrm{~h}$, the cells were digested using trypsin without EDTA and washed twice with pre-cooled PBS. For cell cycle analysis, $2 \times 10^{6}$ cells were fixed in $70 \%$ pre-cooled ethanol (Sigma-Aldrich) and stored in fridge at $-20^{\circ} \mathrm{C}$ overnight. Subsequently, the cells were washed and resuspended 
in PBS, then stained with $500 \mu \mathrm{L}$ propidium iodide (PI) solution (Sigma-Aldrich) containing $100 \mu \mathrm{g} / \mathrm{mL}$ RNase and $50 \mu \mathrm{g} / \mathrm{mL}$ PI in $1 \times$ PBS for $30 \mathrm{~min}$. For cell apoptosis analysis, the cells were harvested according to Annexin V-FITC apoptosis detection kit instructions (BD Biosciences, San Jose, CA, USA). Subsequently, $5 \mu \mathrm{L}$ of PI $(50 \mu \mathrm{g} / \mathrm{mL})$ and $10 \mu \mathrm{L}$ Annexin V-FITC were added into each tube and incubated in the dark for $15 \mathrm{~min}$ at room temperature. Cell cycle and apoptosis rate were analyzed on a BD FACScan (BD Biosciences).

\section{Luciferase reporter assays}

HPA-v cells or 293T cells were seeded in 96-well plates for five repeats each group, then transfected with Lv-AC092159.2 or Lv-NC. After 24h, these cells were transfected with pGL3-Basic TMEM18 (contains TMEM18 promoter sequence) or pGL3-Basic control vector, Lipofectamine 2000, and pRL-TK vector. Cells were cultured in $37^{\circ} \mathrm{C}, 5 \% \mathrm{CO}_{2}$ for $48 \mathrm{~h}$ and relative luciferase activities (ratios of Renilla luciferase signal normalized to firefly luciferase) were determined.

\section{Statistical analysis}

All data were presented as mean \pm s.D. Student's twotailed $t$-test was used to analyze the differences between groups. The Pearson's correlation coefficient analysis was employed to confirm the correlations. Values were considered statistically significant at $P<0.05$.

\section{Results}

\section{The expression characteristics of AC092159.2}

To identify the lncRNAs related to adipogenesis, HPA-v were analyzed for lncRNA expression by lncRNA microarray, and many lncRNAs changed by at least twofold $(P<0.01)$ (data have not been published). LncRNA AC092159.2 was highly expressed in differentiated HPA-v by over threefold compared with undifferentiated HPA-v. Based on GTEx Portal database, AC092159.2 has two isoforms which were expressed in multiple human normal tissues (Fig. 1A and B). However, the isoform expressed in subcutaneous and visceral adipose is mainly ENST00000445418.1, which was used for following research. Change in AC092159.2 was
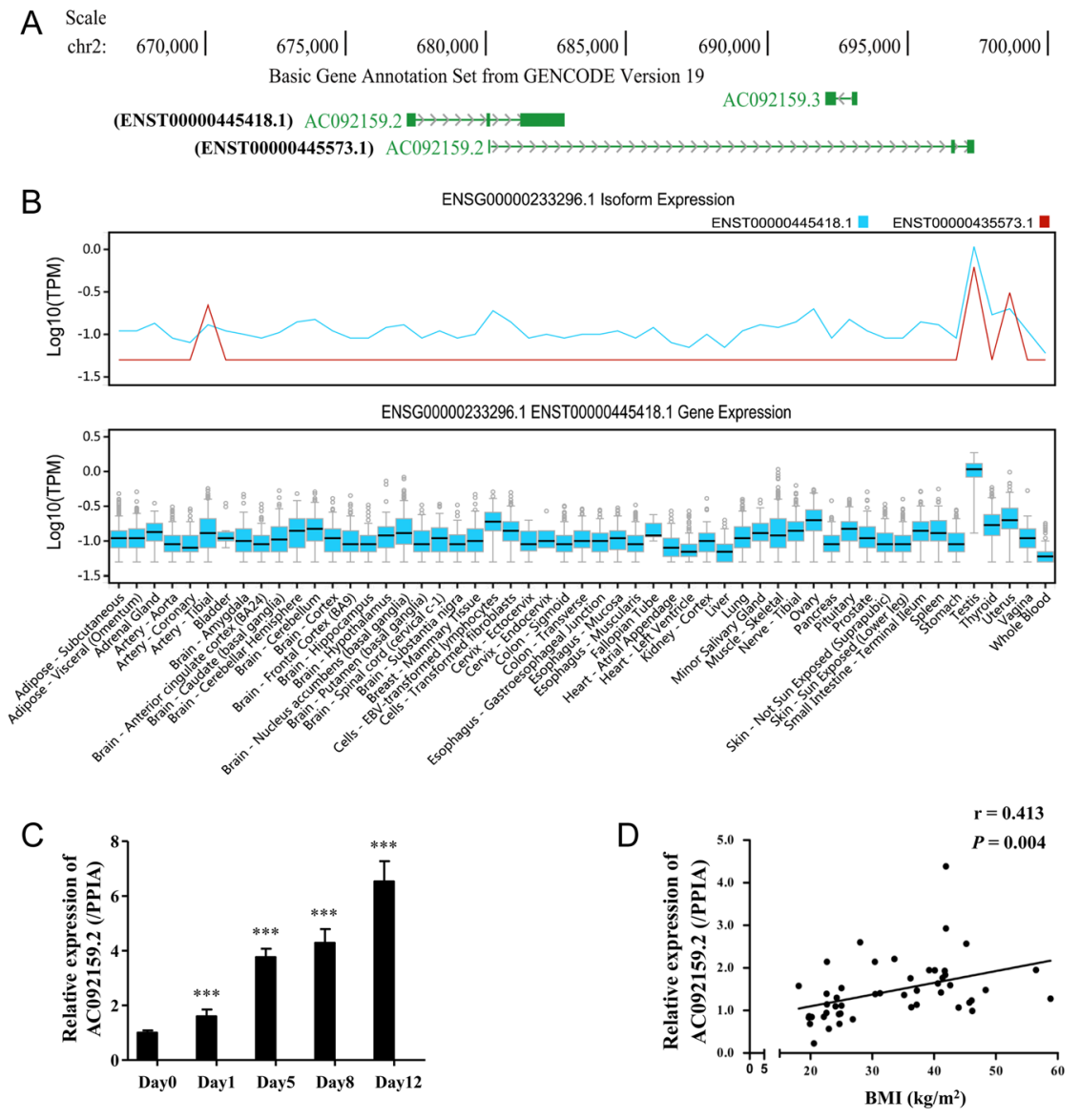

\section{Figure 1}

The expression characteristics of AC092159.2. (A) AC092159.2, also called 'ENSG00000233296.1', has two isoforms ENST00000445418.1 and ENST00000445573.1. (B) AC092159.2 isoform expression in multiple human normal tissues based on GTEx Portal database. The vertical axis shows $\log 10($ TPM). Transcripts per Million (TPM) is an important indicator of gene expression. As shown in the top graph, ENST00000445418.1 was expressed in multiple human normal tissues, whereas ENST00000445573.1 was expressed in only a few tissues. In subcutaneous and visceral adipose, the isoform expressed is mainly ENST00000445418.1. The bottom graph shows ENST00000445418.1 expression in multiple human normal tissues in more detail. (C) Expression of AC092159.2 during HPA-V differentiation was quantitated by qPCR. (D) Positive correlation existed between AC092159.2 levels and BMI among 48 omental adipose tissues of normal and subjects with overweight/obesity. Ratios were calculated as mean \pm S.D. from triplicate samples. Data shown are averages of three independent experiments. ${ }^{*} P<0.05$, $* * P<0.01, * * * P<0.001$. A full color version of this figure is available at https://doi.org/10.1530/ JME-18-0215. 
confirmed by qRT-PCR. The expression of AC092159.2 gradually increased during HPA-v differentiation and peaked at day 12 of differentiation (Fig. 1C). We further evaluated the correlation between AC092159.2 expression in omental adipose tissue and BMI in 31 subjects with overweight/obesity and 17 controls with normal weight. Interestingly, remarkable positive correlation existed between AC092159.2 levels and BMI $(\mathrm{r}=0.413, P=0.004)$ (Fig. 1D). These findings suggested that AC092159.2 may be associated with adipocyte differentiation and obesity.

\section{AC092159.2 promotes human adipocytes differentiation}

To determine whether AC092159.2 affected adipocyte differentiation, HPA-v cells were transduced with a lentivirus expressing AC092159.2 or a mock virus for at least $48 \mathrm{~h}$ before transferring to differentiation medium. Fluorescence microscopy showed that $90 \%$ of cells of the experimental group and the control group were GFP-positive and transduced cells did not emerge any morphological changes. The expression of AC092159.2 was increased 233-fold at day 0 and remained high in the whole process of differentiation (Fig. 2A). Overexpression of AC092159.2 promoted adipocytes differentiation, as indicated by the expression of adipogenesis makers FABP4, PPAR $\gamma, \mathrm{C} / \mathrm{EBP} \beta$ and C/EBP $\alpha$ (Fig. 2B, C, D and E), triacylglycerol content (Fig. 2F) and oil red $\mathrm{O}$ staining (Fig. 2G). In line with qRT-PCR data, the protein levels of FABP4, PPAR $\gamma, \mathrm{C} / \mathrm{EBP} \beta$ and $\mathrm{C} / \mathrm{EBP} \alpha$ were also upregulated after transduction with the AC092159.2-expressing lentivirus (Fig. 2H and I).

The effects of AC092159.2 loss-of-function on adipogenesis were also determined. We performed an
A

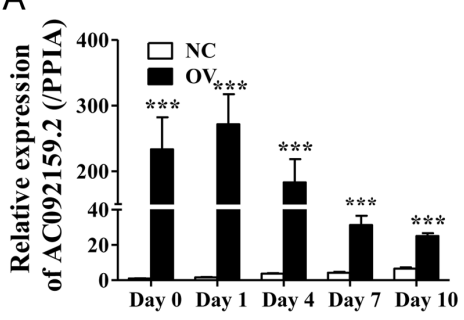

D

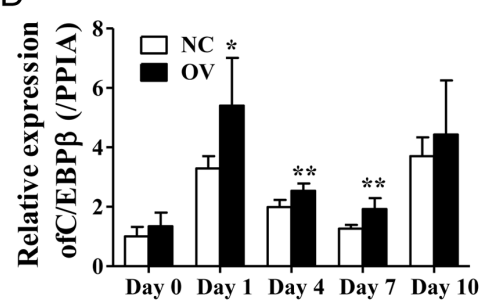

G

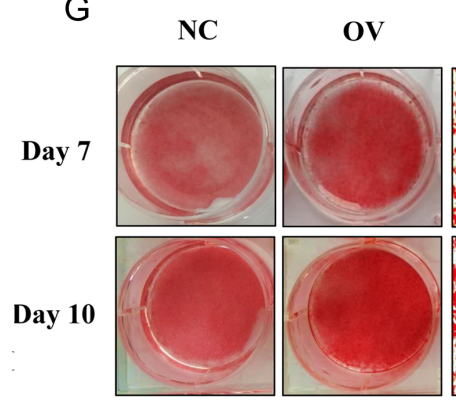

B

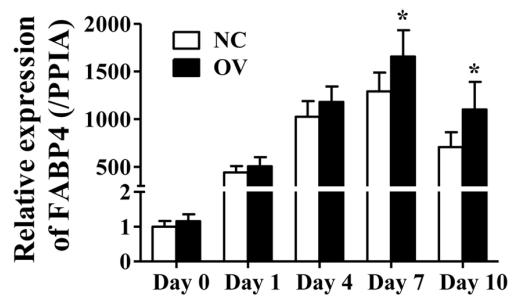

E

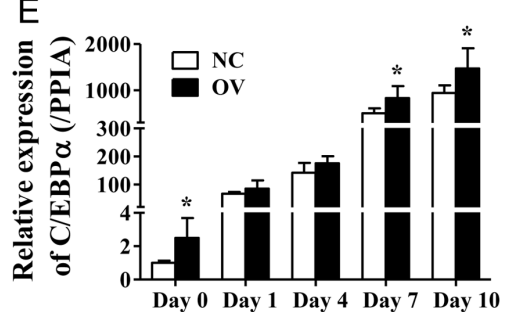

$\mathrm{H}$

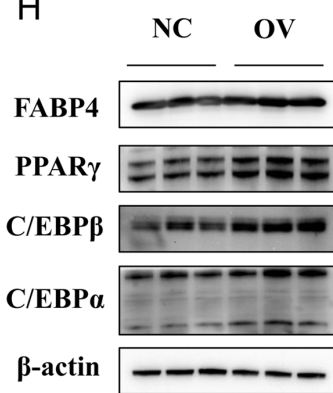

C

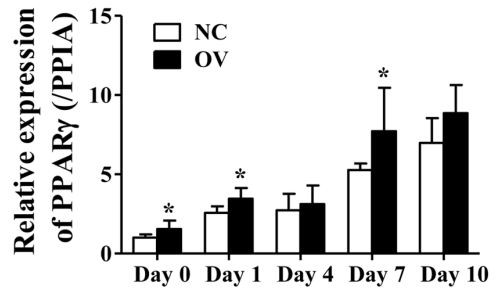

F

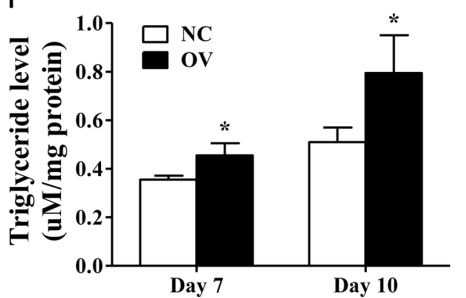

I

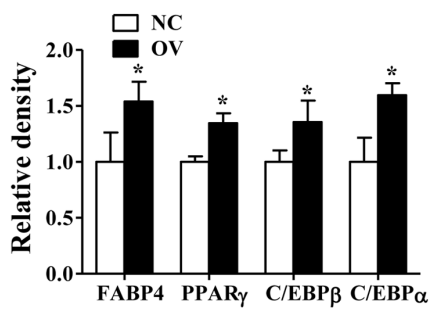

\section{Figure 2}

Overexpression of AC092159.2 in HPA-v promoted adipogenic differentiation. (A) Overexpression efficiency of AC092159.2 was verified by qPCR. (B, C, D and E) AC092159.2 overexpression in HPA-v promoted adipogenic maker gene transcription based on qPCR at days 0, 1, 4, 7 and 10 during adipogenesis. (F) Triacylglycerol content detected neutral lipid accumulation. (G) Oil red O staining indicated the effect of AC092159.2 overexpression on HPA-V adipogenic differentiation at days 7 and 10. $(\mathrm{H})$ Protein levels were detected by Western blot under the same experimental conditions. $(\mathrm{I})$ The quantification of Western blot. Related mRNA levels are represented as mean \pm s.D. from three or more independent repeats. $* P<0.05, \star \star P<0.01$, $\star \star \star P<0.001$. A full color version of this figure is available at https://doi.org/10.1530/JME-18-0215. 
RNAi-knockdown experiment using a pool of siRNAs to transfect them into primary preadipocyte cultures, followed by induction of differentiation. The expression of AC092159.2 was decreased by about 50\% compared with the control (Fig. 3A). At the same time, knockdown of AC092159.2 inhibited adipogenesis, as indicated by reduced adipogenic transcripts FABP4, PPAR $\gamma, \mathrm{C} / \mathrm{EBP} \beta$ and $\mathrm{C} / \mathrm{EBP} \alpha$ (Fig. 3B, C, D and E), triacylglycerol content (Fig. 3F) and Oil Red O staining (Fig. 3G). Likewise, the protein levels of FABP4, PPAR $\gamma, \mathrm{C} / \mathrm{EBP} \beta$ and $\mathrm{C} / \mathrm{EBP} \alpha$ were also decreased (Fig. 3H and I).

\section{AC092159.2 does not influence cell cycle, apoptosis and proliferation}

To further explore other effects on adipocytes performed by AC092159.2, we detected the effect of AC092159.2 on the cell cycle, apoptosis and proliferation. First, we examined the distribution of cells at different cell cycle phases in AC092159.2 transfected HPA-v cells using flow cytometry. Overexpression of AC092159.2 did not significantly influence the cell cycle distribution (Supplementary Fig. $1 \mathrm{~A}$ and B) while knockdown of AC092159.2 slightly decreased the percentage of preadipocytes in the G1 phase and increased the percentage in the $S$ phase, compared with the negative control cells, but the difference was not statistically significant (Supplementary Fig. 1C and D). And we also evaluated the effect of AC092159.2 on the apoptosis of HPA-v cells. The results showed that overexpression of AC092159.2 mildly inhibited the apoptosis of HPA-v cells without any statistical difference (Supplementary Fig. 1E) and AC092159.2 siRNA had no significant effect on HPA-v cells apoptosis (Supplementary Fig. 1F). To further
A

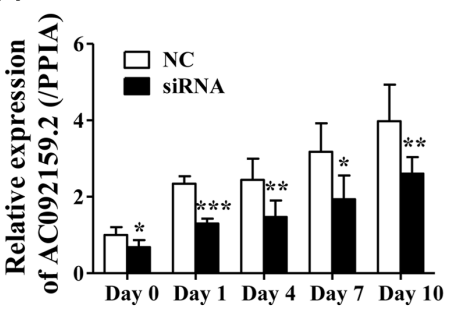

D

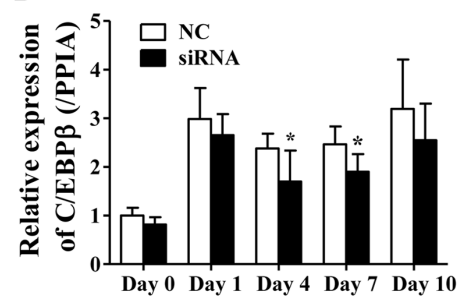

G

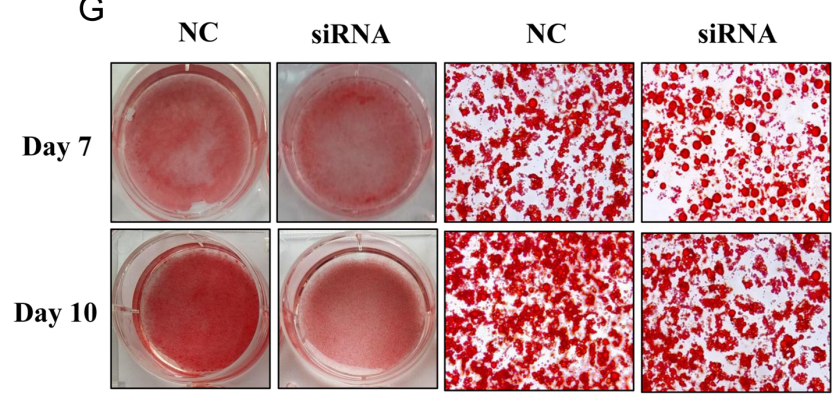

$\mathrm{B}$

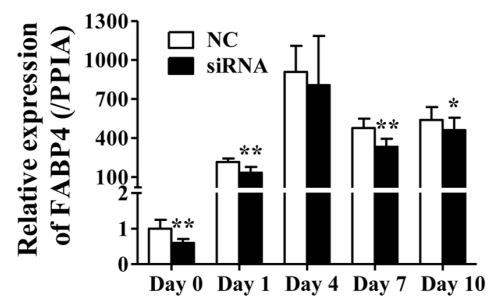

$\mathrm{E}$

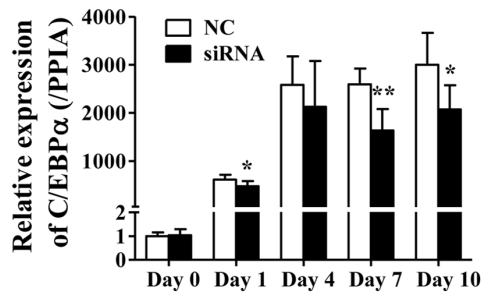

$\mathrm{H}$

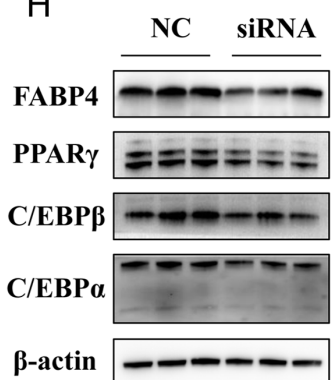

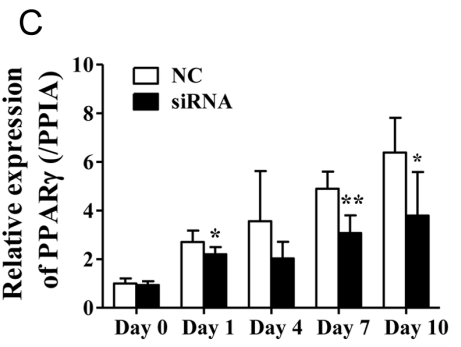

F

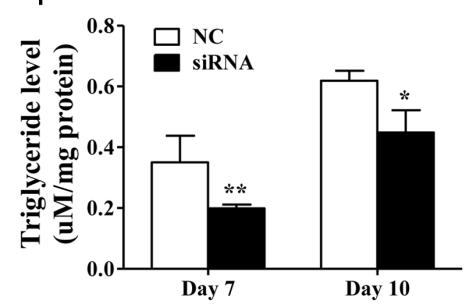

I

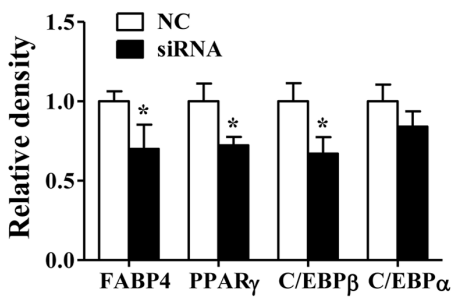

Figure 3

Knockdown of AC092159.2 in HPA-v inhibits adipogenic differentiation. (A) Knockdown efficiency of AC092159.2 was verified by qPCR. (B, C, D and E) Knockdown of AC092159.2 in HPA-v inhibited adipogenic maker gene transcription based on qPCR at days 0, 1, 4, 7 and 10 during adipogenesis. (F) Triacylglycerol content detected neutral lipid accumulation. (G) Oil red O staining indicated the effect of AC092159.2 knockdown on HPA-V adipogenic differentiation at Days 7 and 10. $(\mathrm{H})$ Protein levels were detected by Western blot under the same experimental conditions. (I) The quantification of Western blot. Related mRNA levels are represented as mean \pm s.D. from three or more independent repeats. $* P<0.05, \star \star P<0.01, \star \star \star P<0.001$. A full color version of this figure is available at https://doi.org/10.1530/JME-18-0215. 
evaluate the effect of AC092159.2 on cell proliferation, the CCK-8 assay was used to detect the growth activity of the transfected HPA-v cells in $72 \mathrm{~h}$. At 24,48 and $72 \mathrm{~h}$, both overexpression and knockdown of AC092159.2 had no significant effect on HPA-v cell proliferation compared with control groups (Supplementary Fig. 1G and H). Thus, AC092159.2 is not requisite to maintain HPA-v cell selfrenewal.

\section{The correlation analysis between AC092159.2 and its potential target gene TMEM18}

LncRNAs could modulate expression of genes that are positioned in the vicinity of their transcription sites in a cisacting manner (Song et al. 2012). To further comprehend the mechanism underlying the regulation of adipogenesis by AC092159.2, we searched the transcription position in the UCSC genome browser and found AC092159.2 transcription sites located $\sim 247 \mathrm{bp}$ upstream of the TMEM18 gene (Fig. 4A). Furthermore, based on GTEx Portal database, six single nucleotide polymorphisms (SNPs) in AC092159.2 were associated not only with the expression of AC092159.2, but also with TMEM18, and the effect sizes were similar with consistent direction (Fig. 4B). We further detected the expression of TMEM18 by qPCR among 48 omental adipose tissues of normal and subjects with obesity. The results revealed that the expression of TMEM18 was significantly higher in adipose tissues of subjects with obesity (Fig. 4C) and positively correlated with the expression of AC092159.2 (Fig. 4D). And the expression of TMEM18 and AC092159.2 were also positively correlated during adipogenic differentiation (Fig. 4E). To further verify the relationship between AC092159.2 and TMEM18, we detected the expression of TMEM18 with the overexpression and knockdown of AC092159.2 in HPA-v, respectively. Both qRT-PCR and Western blot revealed that overexpression of AC092159.2 boosted TMEM18 expression while knockdown of AC092159.2 restrained TMEM18 expression (Fig. 4F, G, H and I), suggesting that TMEM18 may be the downstream target gene of AC092159.2.

\section{The effect of AC092159.2 overexpression could be restrained by TMEM18 siRNA in HPA-v during adipogenic differentiation}

The above results had been preliminarily revealed that AC092159.2 participated in adipocyte differentiation possibly by regulating TMEM18. In order to further demonstrate this mechanism, we adopted the rescue strategy. We tested whether the adipogenic bloom induced by overexpression of AC092159.2 could be weakened by knockdown of TMEM18. The results showed that knockdown of TMEM18 partially restrained adipogenic differentiation in AC092159.2 overexpressed HPA-v, as assessed by TMEM18, C/EBP $\beta$ and C/EBP $\alpha$ mRNA (Fig. 5A, $\mathrm{B}$ and $\mathrm{C}$ ), as well as triacylglycerol content (Fig. 5D) and Oil Red O staining (Fig. 5E).

We also knocked down AC092159.2 in TMEM18 overexpressed HPA-v. Adipogenic key genes C/EBP $\beta$ and C/EBP $\propto$ mRNA expression (Fig. 6A, B and C), triacylglycerol content (Fig. 6D) and oil red $\mathrm{O}$ staining (Fig. 6E) all supported that adipogenic defect caused by knockdown of AC092159.2 could be rescued by overexpression of TMEM18. These data revealed that, during adipogenic differentiation, AC092159.2 worked with TMEM18 in HPA-v.

\section{AC092159.2 has a transcriptional activation effect on TMEM18}

The TMEM18 promoter luciferase plasmid pGL3-Basic TMEM18 and Lv-AC092159.2 were cotransfected to HPA-v cells or 293T cells, and the transcriptional activation was detected. The luciferase activity was significantly higher than in the control group after AC092159.2 overexpression and pGL-3-Basic TMEM18 luciferase plasmid transfection for $48 \mathrm{~h}(P<0$ 05; Fig. 7A and B), which indicates that AC092159.2 has a transcriptional activation effect on TMEM18.

\section{Discussion}

In this study, we first reported lncRNA AC092159.2 and found that the expression of AC092159.2 gradually increased during HPA-v differentiation, and its expression in omental adipose tissue was positively related with BMI. Overexpression of AC092159.2 promoted adipocytes differentiation while knockdown of it led to an adipogenic defect. Moreover, the expression of AC092159.2 and TMEM18 were positively correlated during adipogenic differentiation. AC092159.2 overexpression boosted TMEM18 expression while AC092159.2 knockdown restrained TMEM18 expression. Further rescue experiments showed that TMEM18 knockdown partially restrained adipogenic differentiation in AC092159.2 overexpressed HPA-v, and adipogenic defect caused by AC092159.2 knockdown could be rescued by TMEM18 overexpression. Luciferase reporter assays revealed that AC092159.2 had a transcriptional activation effect on TMEM18. 
A

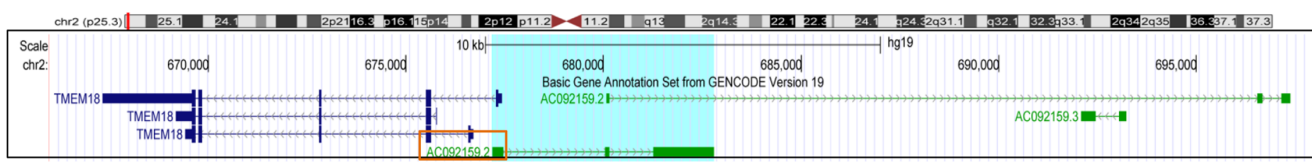

B

\begin{tabular}{ccccccc}
\hline \multirow{2}{*}{ Mapped_gene } & \multirow{2}{*}{ SNP } & \multicolumn{2}{c}{ eQTLs_TMEM18 } & \multicolumn{2}{c}{ eQTLs_AC092159.2 } & \multirow{2}{*}{ Tissue } \\
\cline { 3 - 5 } & & $P$ & Effect size & $P$ & Effect size & \\
\hline AC092159.2 & rs1317961 & $2.7 \mathrm{E}-07$ & 0.30 & $2.6 \mathrm{E}-08$ & 0.34 & Adipose \\
AC092159.2 & rs1320333 & $1.1 \mathrm{E}-09$ & 0.56 & $2.7 \mathrm{E}-08$ & 0.53 & Adipose \\
AC092159.2 & rs2867116 & $2.7 \mathrm{E}-09$ & 0.56 & $7.2 \mathrm{E}-12$ & 0.59 & Adipose \\
AC092159.2 & rs3210390 & $5.7 \mathrm{E}-09$ & 0.63 & $4.8 \mathrm{E}-09$ & 0.54 & Adipose \\
AC092159.2 & rs35181861 & $2.6 \mathrm{E}-19$ & 0.73 & $2.4 \mathrm{E}-12$ & 0.60 & Adipose \\
AC092159.2 & rs4241325 & 3.9E-12 & -0.43 & $1.5 \mathrm{E}-14$ & -0.48 & Adipose \\
\hline
\end{tabular}

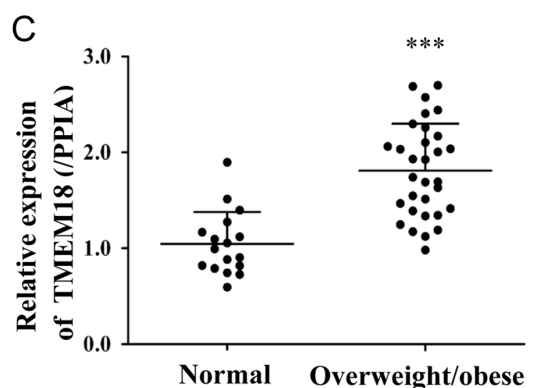

$\mathrm{F}$

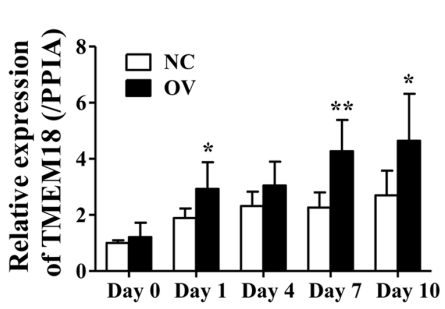

D

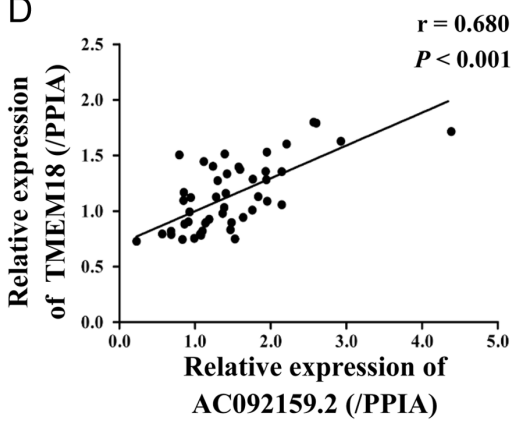

E

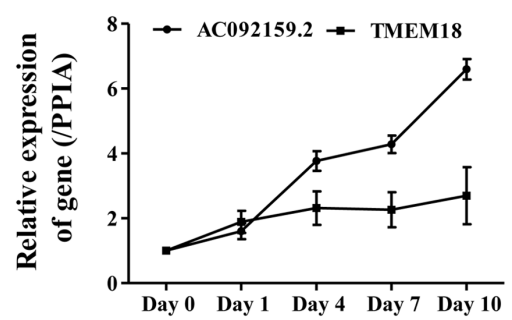

G

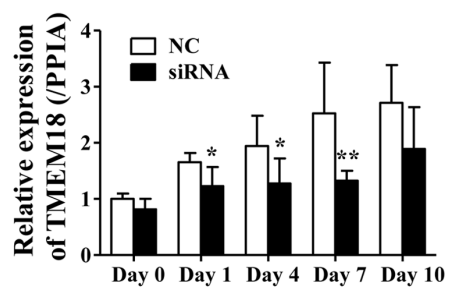

$\mathrm{H}$

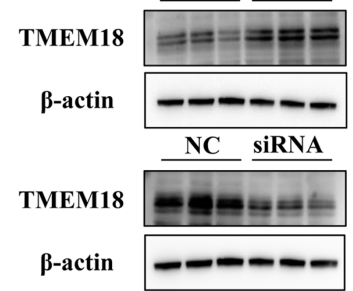

I

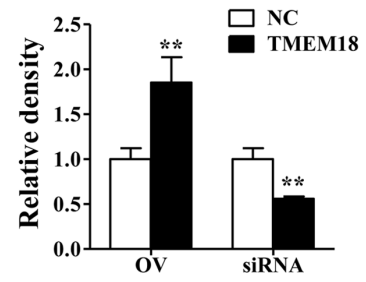

Figure 4

The correlation analysis between AC092159.2 and its potential target gene TMEM18. (A) The obesity susceptibility gene TMEM18 is adjacent to AC092159.2. (B) Based on GTEx Portal database, six SNPs in AC092159.2 were associated not only with the expression of AC092159.2, but also with TMEM18. And the effect sizes were similar with consistent direction. (C) Relative expression of TMEM18 by qPCR among 48 omental adipose tissues of normal and subjects with overweight/obesity. Ratios were calculated as mean \pm s.D. (D) Positive correlation existed between TMEM18 levels and BMI among the 48 omental adipose tissues of normal and subjects with obesity. (E) Relative expression of AC092159.2 and TMEM18 during HPA-v differentiation was quantitated by qPCR. Ratios were calculated as mean \pm s.D. from triplicate samples. $(F, G$ and $H)$ The relative expression of TMEM18 with the overexpression and knockdown of AC092159.2 in HPA-v, respectively. (I) The quantification of Western blot. Data shown are averages of three independent experiments. $\star P<0.05, * \star P<0.01, * * \star P<0.001$. A full color version of this figure is available at https://doi.org/10.1530/JME-18-0215.

Therefore, AC092159.2 may serve as a positive regulator of adipocyte differentiation, and promoted the adipogenesis of human preadipocyte possibly by regulating TMEM18. It is indicated that AC092159.2 may play an essential role in obesity and related metabolic disorders. However, the precise molecular network that underlies regulation of adipocyte differentiation is still unclear, so further efforts to confirm the regulatory factors involved are of great concern. Further exploring the role and mechanism of AC092159.2 in adipocyte differentiation will ultimately open a new avenue to understand adipogenesis.
LncRNAs have vital roles in regulating cell fate during developmental and differentiation processes and are often lineage-specific in multicellular organisms (Batista \& Chang 2013). Multiple IncRNAs have been identified to be involved in adipocyte differentiation (Kikuchi et al. 2009, Sun et al. 2013, Xiao et al. 2015) and lncRNAs were reported to regulate the expression of adjacent genes as cisacting regulatory elements (Ponting et al. 2009). LncRNA ADINR transcribed from a position $\sim 450 \mathrm{bp}$ upstream of the $C / E B P \alpha$ gene, orchestrates $\mathrm{C} / \mathrm{EBP} \alpha$ transcription in vivo. It is shown that ADINR plays an important role 
A

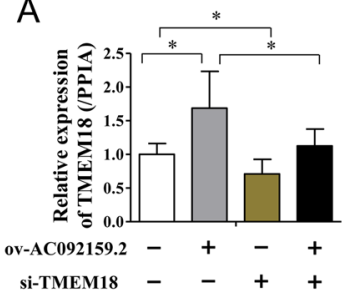

D

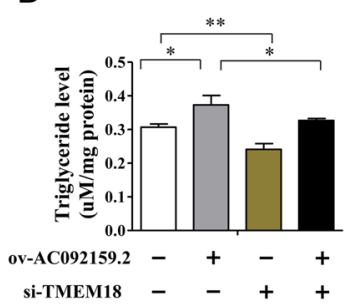

B

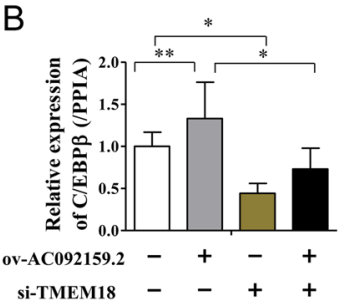

C

E

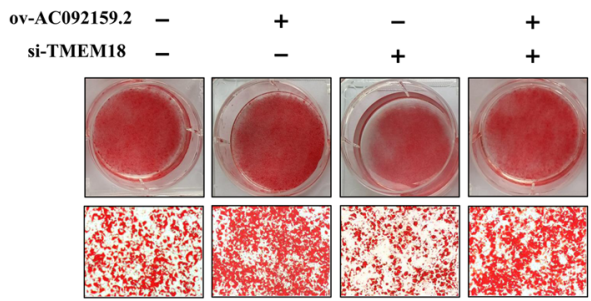

Figure 5

The effect of AC092159.2 overexpression could be rescued by TMEM18 siRNA in HPA-v during adipogenic differentiation. (A, B and C) qPCR analysis of adipogenic maker genes (C/EBP $\beta$ and C/EBP $\alpha$ ) on day 7 of adipogenic differentiation. (D) Triacylglycerol content detected neutral lipid accumulation on day 7 of adipogenic differentiation. (E) Oil red O staining on day 7 of adipogenic differentiation. Related mRNA levels are represented as mean \pm s.D. from three or more independent repeats. ${ }^{*} P<0.05, * * P<0.01$, $* \star * P<0.001$. A full color version of this figure is available at https://doi.org/10.1530/JME-18-0215. in adipogenesis through regulating $\mathrm{C} / \mathrm{EBP} \alpha$ in cis (Xiao et al. 2015). LncRNA U90926 was reported to attenuate 3T3-L1 adipocyte differentiation via inhibiting the transactivation of PPAR $\gamma 2$ or PPAR $\gamma$ (Chen et al. 2017). It has been confirmed that the transcription of most lncRNAs are in coordination with transcription of proteincoding genes and over 2000 lncRNAs are bidirectionally transcribed within $2 \mid \mathrm{kb}$ of the transcription start sites of protein-coding genes (Sigova et al. 2013). Our findings suggested that AC092159.2 exerted its effect possibly by impacting on its neighboring gene TMEM18 which has been confirmed as an obesity susceptibility gene (Den Hoed et al. 2010, Jurvansuu \& Goldman 2011, Berndt et al. 2013, Wheeler et al. 2013, Locke et al. 2015, Larder et al. 2017).

TMEM18 is a transmembrane protein of 140 amino acids containing four transmembrane domains which could act as DNA-binding protein for other downstream genes (Jurvansuu \& Goldman 2011) and plausible mediator of genetic variation on human adiposity (Larder et al. 2017). In recent years, researches have confirmed that TMEM18 was an obesity-associated gene (Den Hoed et al. 2010, Berndt et al. 2013, Wheeler et al. 2013, Locke et al. 2015, Larder et al. 2017) and TMEM18 was identified to be crucial for adipogenesis (Bernhard et al. 2013). Moreover, the methylation levels of TMEM18 promoter were possibly associated with parameters of obesity and fat distribution (Rohde et al. 2014). Similar to our study, mRNA expression of TMEM18 was reported to be well correlated with body weight in rats (Rask-Andersen et al. 2012) and knockdown of TMEM18 resulted in an obvious reduction in triacylglycerol accumulation and adipocyte differentiation in HPA-v (Bernhard et al. 2013). However, Rachel et al. reported that loss of Tmem18 in male mice
A

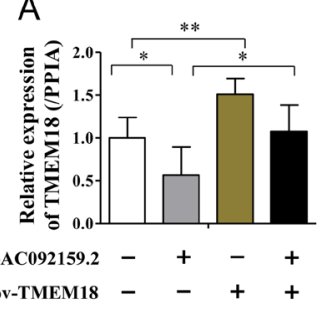

D

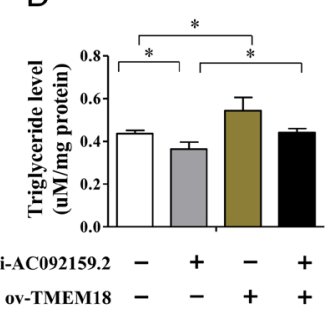

B

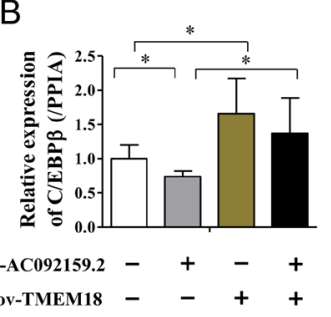

C

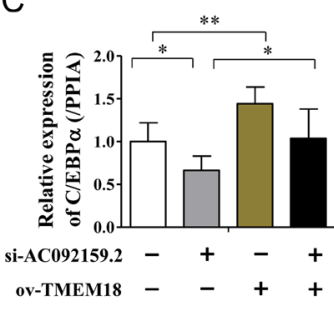

\section{Figure 6}

The effect of AC092159.2 siRNA could be rescued by TMEM18 overexpression in HPA-v during adipogenic differentiation. (A, B and C) qPCR analysis of adipogenic maker genes (C/EBP $\beta$ and C/EBP $\alpha$ ) on day 7 of adipogenic differentiation. (D) Triacylglycerol content detected neutral lipid accumulation on day 7 of adipogenic differentiation. (E) Oil red $\mathrm{O}$ staining on day 7 of adipogenic differentiation. Related mRNA levels are represented as mean \pm S.D. from three or more independent repeats. $* P<0.05, * * P<0.01$, $\star \star \star P<0.001$. A full color version of this figure is available at https://doi.org/10.1530/JME-18-0215. 
A
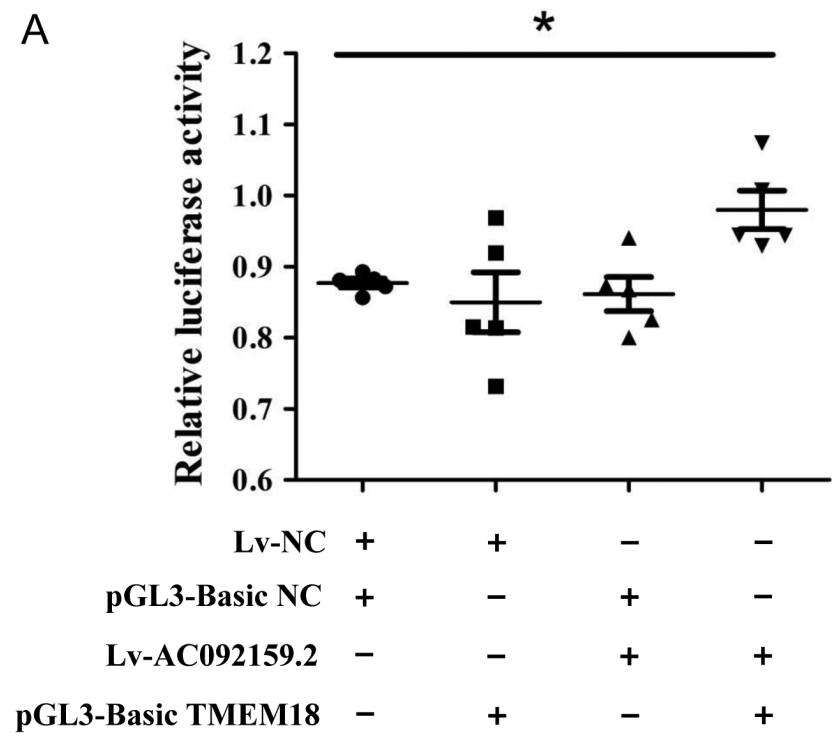

B

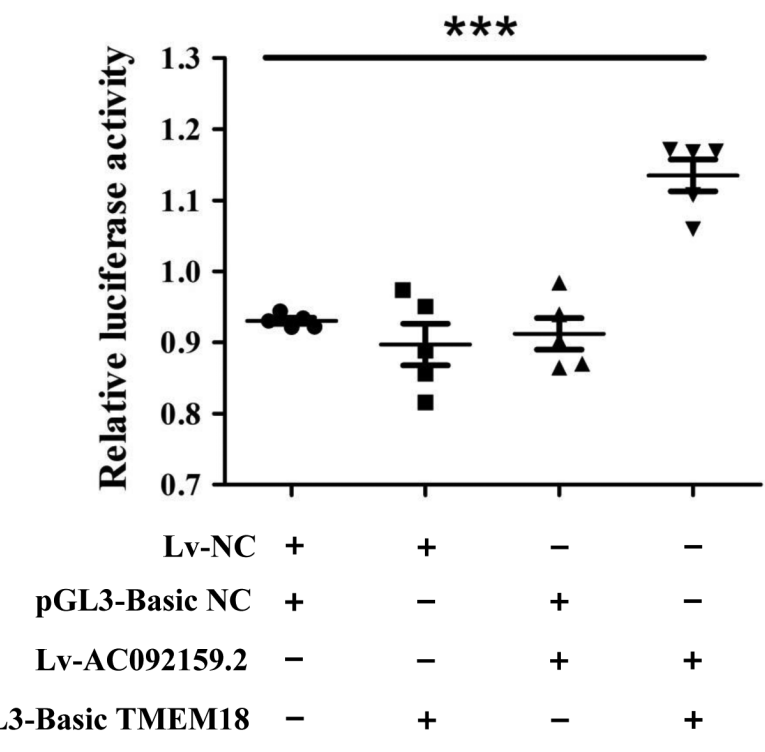

Figure 7

Luciferase reporter assays show that transcriptional activation of TMEM18 promoter in response to AC092159.2 overexpression. (A) In HPA-v. (B) In $293 \mathrm{~T}$ cells. Related luciferase activities are from five repeats each group. $\star P<0.05, * * P<0.01, * * * P<0.001$.

led to an increase in body weight, which was aggravated by high-fat diet and influenced by increased food intake. Overexpression of Tmem18 in the hypothalamic paraventricular nucleus (PVN) of wild-type mice led to decreased food intake and increased energy expenditure (Larder et al. 2017). But the phenotype of increased body weight in Tmem18 null mice was sexually dimorphic (Larder et al. 2017). In the above research, TMEM18 exerted its effect on metabolism in PVN mainly by affecting appetite (Larder et al. 2017). It is shown that the effect of TMEM18 on metabolic regulation may be tissueand gender-specific. The tissues specificity and potential biological mechanisms relevant to this phenomenon are still controversial (Kozak 2010). So, further tissue-specific and sex-specific method may prove more productive. Although the precise molecular mechanism that underlies regulation of TMEM18 is not fully understood, we identified that TMEM18 may be the target gene of AC092159.2 and AC092159.2 may exert its function by acting on TMEM18.

\section{Conclusion}

Taken together, we identified AC092159.2 as an uncharacteristic transcriptional regulator of TMEM18 and concluded that AC092159.2 participated in the development of adipogenesis by interacting with TMEM18, which shed new light onto how lncRNAs harmonizes the regulation of downstream gene expression and adipogenic differentiation. Thus, further understanding the definite role of lncRNAs in gene regulatory networks and diseases may provide a novel potential target for the development of human therapeutics.

Supplementary data

This is linked to the online version of the paper at https://doi.org/10.1530/ JME-18-0215.

\section{Declaration of interest}

The authors declare that there is no conflict of interest that could be perceived as prejudicing the impartiality of the research reported.

\section{Funding}

This work was supported in part by the National Natural Science Foundation of China $(81600685,81600687,81770866,81770837,81330067,81670773$, 81500649, 81500674), Jiangsu Province Natural Science Foundation (BK20160141, BK20150082, BK20140086), Nanjing Medical Science and Technique Development Foundation (YKK16201, QRX17163, YKK15160, YKK15164, QRX17160), 333 high-level talents training project of Jiangsu Province, Six talent peaks project in Jiangsu Province (WSN-121) and Jiangsu Provincial Medical Youth Talent (QNRC2016108, QNRC2016109).

\section{Author contribution statement}

Juan Wen and Xirong Guo designed the research. Yingdi Yuan, Xinguo Cao, Jiaojiao Hu, Jingyun Li, Dan Shen, Lianghui You, Xing Wang, Yahui Zhou, Yao Gao, Lijun Zhu and Pengfei Xu performed the experiments. Lianghui You and Xing Wang analyzed the data. Yingdi Yuan and Juan Wen wrote this manuscript. Chenbo Ji supervised this work. All the authors reviewed the report and approved the final version. 


\section{References}

Barbosa-Desongles A, Hernandez C, Simo R \& Selva DM 2013 Testosterone induces cell proliferation and cell cycle gene overexpression in human visceral preadipocytes. American Journal of Physiology Cell Physiology 305 C355-C359. (https://doi.org/10.1152/ ajpcell.00019.2013)

Barth RJ 2011 Insulin resistance, obesity and the metabolic syndrome. South Dakota Medicine Spec No 22-27.

Batista PJ \& Chang HY 2013 Long noncoding RNAs: cellular address codes in development and disease. Cell 152 1298-1307. (https://doi. org/10.1016/j.cell.2013.02.012

Berndt SI, Gustafsson S, Magi R, Ganna A, Wheeler E, Feitosa MF, Justice AE, Monda KL, Croteau-Chonka DC, Day FR, et al. 2013 Genome-wide meta-analysis identifies 11 new loci for anthropometric traits and provides insights into genetic architecture. Nature Genetics 45 501-512. (https://doi.org/10.1038/ng.2606)

Bernhard F, Landgraf K, Kloting N, Berthold A, Buttner P, Friebe D, Kiess W, Kovacs P, Bluher M \& Korner A 2013 Functional relevance of genes implicated by obesity genome-wide association study signals for human adipocyte biology. Diabetologia 56 311-322. (https://doi.org/10.1007/s00125-012-2773-0)

Chen J, Liu Y, Lu S, Yin L, Zong C, Cui S, Qin D, Yang Y, Guan Q, Li X, et al. 2017 The role and possible mechanism of lncRNA U90926 in modulating 3T3-L1 preadipocyte differentiation. International Journal of Obesity 41 299-308. (https://doi.org/10.1038/ijo.2016.189)

Cristancho AG \& Lazar MA 2011 Forming functional fat: a growing understanding of adipocyte differentiation. Nature Reviews Molecular Cell Biology 12 722-734. (https://doi.org/10.1038/nrm3198)

Cui X, You L, Li Y, Zhu L, Zhang F, Xie K, Cao Y, Ji C \& Guo X 2016 A transcribed ultraconserved noncoding RNA, uc.417, serves as a negative regulator of brown adipose tissue thermogenesis. FASEB Journal 30 4301-4312. (https://doi.org/10.1096/fj.201600694R)

Davidge-Pitts C, Escande CJ \& Conover CA 2014 Preferential expression of PAPPA in human preadipocytes from omental fat. Journal of Endocrinology 222 87-97. (https://doi.org/10.1530/JOE-13-0610)

Den Hoed M, Ekelund U, Brage S, Grontved A, Zhao JH, Sharp SJ, Ong KK, Wareham NJ \& Loos RJ 2010 Genetic susceptibility to obesity and related traits in childhood and adolescence: influence of loci identified by genome-wide association studies. Diabetes 59 2980-2988. (https://doi.org/10.2337/db10-0370)

Farmer SR 2006 Transcriptional control of adipocyte formation. Cell Metabolism 4 263-273. (https://doi.org/10.1016/j.cmet.2006.07.001)

Fujita K, Iwama H, Oura K, Tadokoro T, Hirose K, Watanabe M, Sakamoto T, Katsura A, Mimura S, Nomura T, et al. 2016 Metforminsuppressed differentiation of human visceral preadipocytes: involvement of microRNAs. International Journal of Molecular Medicine 38 1135-1140. (https://doi.org/10.3892/ijmm.2016.2729)

Gupta RA, Shah N, Wang KC, Kim J, Horlings HM, Wong DJ, Tsai MC, Hung T, Argani P, Rinn JL, et al. 2010 Long non-coding RNA HOTAIR reprograms chromatin state to promote cancer metastasis. Nature 464 1071-1076. (https://doi.org/10.1038/nature08975)

Haslam DW \& James WP 2005 Obesity. Lancet 366 1197-1209. (https:// doi.org/10.1016/S0140-6736(05)67483-1)

Hu W, Alvarez-Dominguez JR \& Lodish HF 2012 Regulation of mammalian cell differentiation by long non-coding RNAs. EMBO Reports 13 971-983. (https://doi.org/10.1038/embor.2012.145)

Huang Y, Zheng Y, Jin C, Li X, Jia L \& Li W 2016 Long non-coding RNA H19 inhibits adipocyte differentiation of bone marrow mesenchymal stem cells through epigenetic modulation of histone deacetylases. Scientific Reports 6 28897. (https://doi.org/10.1038/srep28897)

Jurvansuu JM \& Goldman A 2011 Obesity risk gene TMEM18 encodes a sequence-specific DNA-binding protein. PLoS One 6 e25317. (https:// doi.org/10.1371/journal.pone.0025317)

Kikuchi K, Fukuda M, Ito T, Inoue M, Yokoi T, Chiku S, Mitsuyama T, Asai K, Hirose T \& Aizawa Y 2009 Transcripts of unknown function in multiple-signaling pathways involved in human stem cell differentiation. Nucleic Acids Research 37 4987-5000. (https://doi. org/10.1093/nar/gkp426)

Kornfeld JW \& Bruning JC 2014 Regulation of metabolism by long, noncoding RNAs. Frontiers in Genetics 5 57. (https://doi.org/10.3389/ fgene.2014.00057)

Kozak LP 2010 Brown fat and the myth of diet-induced thermogenesis. Cell Metabolism 11 263-267. (https://doi.org/10.1016/j. cmet.2010.03.009)

Larder R, Sim MFM, Gulati P, Antrobus R, Tung YCL, Rimmington D, Ayuso E, Polex-Wolf J, Lam BYH, Dias C, et al. 2017 Obesityassociated gene TMEM18 has a role in the central control of appetite and body weight regulation. PNAS 114 9421-9426. (https://doi. org/10.1073/pnas.1707310114)

Lee JT 2009 Lessons from X-chromosome inactivation: long ncRNA as guides and tethers to the epigenome. Genes and Development 23 1831-1842. (https://doi.org/10.1101/gad.1811209)

Lefterova MI \& Lazar MA 2009 New developments in adipogenesis. Trends in Endocrinology and Metabolism 20 107-114. (https://doi. org/10.1016/j.tem.2008.11.005)

Locke AE, Kahali B, Berndt SI, Justice AE, Pers TH, Day FR, Powell C, Vedantam S, Buchkovich ML, Yang J, et al. 2015 Genetic studies of body mass index yield new insights for obesity biology. Nature $\mathbf{5 1 8}$ 197-206. (https://doi.org/10.1038/nature14177)

Lu S, Guan Q, Liu Y, Wang H, Xu W, Li X, Fu Y, Gao L, Zhao J \& Wang X 2012 Role of extrathyroidal TSHR expression in adipocyte differentiation and its association with obesity. Lipids in Health and Disease 11 17. (https://doi.org/10.1186/1476-511X-11-17)

Ng M, Fleming T, Robinson M, Thomson B, Graetz N, Margono C, Mullany EC, Biryukov S, Abbafati C, Abera SF, et al. 2014 Global, regional, and national prevalence of overweight and obesity in children and adults during 1980-2013: a systematic analysis for the Global Burden of Disease Study 2013. Lancet 384 766-781. (https:// doi.org/10.1016/S0140-6736(14)60460-8)

Ponting CP, Oliver PL \& Reik W 2009 Evolution and functions of long noncoding RNAs. Cell 136 629-641. (https://doi.org/10.1016/j. cell.2009.02.006

Rask-Andersen M, Jacobsson JA, Moschonis G, Chavan RA, Sikder MA, Allzen E, Alsio J, Chrousos GP, Manios Y, Fredriksson R, et al. 2012 Association of TMEM18 variants with BMI and waist circumference in children and correlation of mRNA expression in the PFC with body weight in rats. European Journal of Human Genetics 20 192-197. (https://doi.org/10.1038/ejhg.2011.176)

Rinn JL \& Chang HY 2012 Genome regulation by long noncoding RNAs. Annual Review of Biochemistry 81 145-166. (https://doi. org/10.1146/annurev-biochem-051410-092902)

Rohde K, Keller M, Klos M, Schleinitz D, Dietrich A, Schon MR, Gartner D, Lohmann T, Dressler M, Stumvoll M, et al. 2014 Adipose tissue depot specific promoter methylation of TMEM18. Journal of Molecular Medicine 92 881-888. (http://doi.org/10.1007/s00109-0141154-1)

Serra-Majem L \& Bautista-Castano I 2013 Etiology of obesity: two "key issues" and other emerging factors. Nutricion Hospitalaria $\mathbf{2 8}$ (Supplement 5) 32-43. (https://doi.org/10.3305/nh.2013.28. sup5.6916)

Sigova AA, Mullen AC, Molinie B, Gupta S, Orlando DA, Guenther MG Almada AE, Lin C, Sharp PA, Giallourakis CC, et al. 2013 Divergent transcription of long noncoding RNA/mRNA gene pairs in embryonic stem cells. PNAS 110 2876-2881. (https://doi. org/10.1073/pnas.1221904110)

Song X, Wang X, Arai S \& Kurokawa R 2012 Promoter-associated noncoding RNA from the CCND1 promoter. Methods in Molecular Biology 809 609-622. (https://doi.org/10.1007/978-1-61779-3769_39)

Sun L, Goff LA, Trapnell C, Alexander R, Lo KA, Hacisuleyman E, Sauvageau M, Tazon-Vega B, Kelley DR, Hendrickson DG, et al. 2013 
Long noncoding RNAs regulate adipogenesis. PNAS 110 3387-3392. (https://doi.org/10.1073/pnas.1222643110)

Tsai MC, Manor O, Wan Y, Mosammaparast N, Wang JK, Lan F, Shi Y, Segal E \& Chang HY 2010 Long noncoding RNA as modular scaffold of histone modification complexes. Science 329 689-693. (https:// doi.org/10.1126/science.1192002)

Wang H, Du S, Zhai F \& Popkin BM 2007 Trends in the distribution of body mass index among Chinese adults, aged 20-45 years (19892000). International Journal of Obesity 31 272-278. (https://doi. org/10.1038/sj.ijo.0803416)

Wheeler E, Huang N, Bochukova EG, Keogh JM, Lindsay S, Garg S, Henning E, Blackburn H, Loos RJ, Wareham NJ, et al. 2013 Genomewide SNP and CNV analysis identifies common and low-frequency variants associated with severe early-onset obesity. Nature Genetics $\mathbf{4 5}$ 513-517. (https://doi.org/10.1038/ng.2607)

Xiao T, Liu L, Li H, Sun Y, Luo H, Li T, Wang S, Dalton S, Zhao RC \& Chen R 2015 Long noncoding RNA ADINR regulates adipogenesis by transcriptionally activating C/EBPalpha. Stem Cell Reports 5 856-865. (https://doi.org/10.1016/j.stemcr.2015.09.007)

Yang L, Li P, Yang W, Ruan X, Kiesewetter K, Zhu J \& Cao H 2016 Integrative transcriptome analyses of metabolic responses in mice define pivotal LncRNA metabolic regulators. Cell Metabolism 24 627-639. (https://doi.org/10.1016/j.cmet.2016.08.019)

Zhao XY \& Lin JD 2015 Long noncoding RNAs: a new regulatory code in metabolic control. Trends in Biochemical Sciences 40 586-596. (https://doi.org/10.1016/j.tibs.2015.08.002)

Received in final form 11 January 2019

Accepted 11 February 2019

Accepted Preprint published online 11 February 2019
(C) 2019 Society for Endocrinology Published by Bioscientifica Ltd. Printed in Great Britain 